\title{
On The Equivariant Isotopy Classes of Some Equivariant Imbeddings of Spheres
}

By

\section{Kōjun $\mathrm{ABE}^{*}$}

\section{§ $0 . \quad$ Introduction}

The purpose of this paper is to study the $G$-isotopy classes of $G$-imbeddings of spheres into spheres, where the spheres are equipped with semi-free linear $G$-actions for a finite group $G$.

Let $V$ be an $m$-dimensional real $G$-module. Throughout this paper we shall assume that $V$ is a product module $V=R^{n} \oplus V_{1}$ of a trivial real $G$-module $R^{n}$ of positive dimension $n$ and an $(m-n)$ dimensional real $G$-module $V_{1}$ on the $G$-invariant unit sphere $S\left(V_{1}\right)$ of which $G$ acts freely. Let $W$ be a real $G$-module which contains $V$ as a direct summand. Let $S^{v}$ and $S^{w}$ denote the one-point compactifications of $V$ and $W$ respectively. Then $S^{v}$ and $S^{w}$ are spheres on which $G$ acts linearly. The direct sum of $d$ copies of $V$ will be denoted by $d V$.

Theorem A. Let $G$ be a cyclic group $Z_{q}$ and let $W=d V \oplus R^{k}$ for $k>m+1$. If $d \geqq \max \{(n+3) / 2,(m+2) /(m-n)\}$, then any $G$ imbedding of $S^{V}$ into $S^{W}$ is $G$-isotopic to the standard imbedding.

Theorem B. Let $G$ be a cyclic group $Z_{q}$ for $q>2$ and let $W=d V \oplus R^{k}$ for $k>m+1$. Suppose that $d \geqq(m+1) /(m-n)$ and $V_{1}$ is a direct sum of $(m-n) / 2$ copies of an irreducible 2-dimensional real $G$-module.

(1) If $d=(m+1) /(m-n)$, then there are infinitely many $G$ -

* Department of Mathematics, Shinshu University, Matsumoto, 390, Japan. 
imbeddings of $S^{v}$ into $S^{\mathrm{W}}$ which are not G-isotopic to each other, and (2) if $d>(m+1) /(m-n)$, then any $G$-imbedding of $S^{\mathrm{V}}$ into $S^{\mathrm{w}}$ is $G$-isotopic to the standard imbedding.

The paper is organized as follows. For any $G$-imbedding $f: S^{v}$ $\rightarrow S^{w}$, we shall show that, by $G$-isotopies, $f \mid S^{n}$ can be deformed to be standard in $\S 1, f$ can be deformed to be linear on a neighborhood of $S^{n}$ in $\S 2$ and $f$ can be deformed to be orthogonal on a neighborhood of $S^{n}$ in $\S 3$. Moreover we shall prove that, if two $G$-imbeddings of $S^{v}$ into $S^{\text {w }}$ are $G$-isotopic and are orthogonal on a neighborhood of $S^{n}$, then there exists a $G$-isotopy between them which is orthogonal on a neighborhood of $S^{n}$ in $\S 3$. Then we see that the $G$-isotopy class of $f$ is determined by the homotopy class of the orbit map of $f \mid\left(S^{v}-U\right)$ relative to the boundary, where $U$ is a neighborhood of $S^{n}$. In $\S 4$, using the obstruction theory, we shall prove Theorem A and Theorem B.

The author wishes to thank Professors M. Adachi and T. Matumoto for their kind advices and valuable criticism.

\section{§ 1. Imbeddings Can Be Deformed to Be Standard on the Fixed Point Set}

In this paper we shall assume that all manifolds and all actions are differentiable of class $C^{\infty}$. Until Section 3 the results are valid in the case of $G$ a compact Lie group.

In this section we shall prove that any $G$-imbedding of $S^{v}$ into $S^{\mathrm{w}}$ is $G$-isotopic to a $G$-imbedding which is standard on $S^{n}$ (see Proposition 1.3), and if two $G$-imbeddings of $S^{v}$ into $S^{w}$, which are standard on $S^{n}$, are $G$-isotopic, then there exists a $G$-isotopy between them which is standard on $S^{n}$ (see Proposition 1.4).

Definition 1. 1. Let $M$ be a $G$-submanifold of a $G$-manifold N. Let $I$ denote the unit interval $[0,1]$ with trivial $G$-action. A smooth map (resp. smooth $G$-map) $f: M \times I \rightarrow N$ is said to be an isotopy (resp. $G$-isotopy) if each $f_{t}: M \rightarrow N$ is an imbedding (resp. $G$-imbed- 
ding), where $f_{t}(x)=f(x, t)$, and $f_{t}$ is independent of $t$ in some neighborhood of 0 and in some neighorhood of 1 (see G. Bredon [1, Chapter VI, §3]). Two imbeddings (resp. $G$-imbeddings) $f_{i}: M$ $\rightarrow N(i=0,1)$ are said to be isotopic (resp. $G$-isotopic) if there exists an isotopy (resp. $G$-isotopy) $\mathrm{F}: M \times I \rightarrow N$ with $F_{0}=f_{0}$ and $F_{1}=f_{1}$. If $\partial M$ is not empty, we shall consider $M \times I$ as a smooth manifold with corners.

Let $I\left(S^{v}, S^{\mathrm{w}}\right)$ denote the set of all $G$-isotopy classes of $G$-imbeddings $f: S^{\mathrm{v}} \rightarrow S^{\mathrm{w}}$. Our purpose is to determine the set of $I\left(S^{\mathrm{v}}, S^{\mathrm{w}}\right)$, provided that $W=d V \oplus R^{k}$ for $k>m+1$.

Remarks. 1. It is easy to see that any $G$-map $f: S^{v} \rightarrow S^{W}$ is $G$ homotopic to the standard imbedding.

2. Using the method of A. Wasserman $[7, \S 1]$, we can see that any $G$-imbedding $f: S^{v} \rightarrow S^{W}$ is $G$-isotopic to the standard imbedding if $d>2 m+2$.

The following lemma will be useful.

Lemma 1.2. Let $N$ be a q-dimensional manifold on which $G$ acts semi-freely and let $M$ be a p-dimensional G-submanifold of $N$. Let $K$ denote $I$ or $I \times I$ and let $L$ be a closed subset of $K$ which contains $\partial K$. Let $f: M \times K \rightarrow N$ be a continuous $G$-map such that each $f_{k}: M \rightarrow N$ is a G-imbedding, where $f_{k}$ is defined by $f_{k}(x)=f(x, k)$. If $f$ is a smooth G-map on $M \times L^{\prime}$, where $L^{\prime}$ is a neighborhood of $L$ in $K$, then there exists a smooth G-map $H: M \times K \rightarrow N$ such that each $H_{k}$ is a G-imbedding and $H=f$ on $M \times L$, where $H_{k}: M \rightarrow N$ is defined by $H_{k}(x)=H(x, k)$.

Proof. We shall prove Lemma 1.2 by an equivariant version of J. Munkres' argument [4, Chapter I, §4]. Let $\left\{U_{i}\right\}$ (resp. $\left\{V_{j}\right\}$ ) be a family of locally finite countable invariant open sets of $M$ such that $\bigcup_{i} U_{i} \subset M^{G}$ (resp. $\bigcup_{j} V_{j} \supset N^{G}$ ) and $\bar{U}_{i}$ (resp. $V_{j}$ ) is equivariant diffeomorphic to a $p$-dimensional disc or half disc (resp. $q$-dimensional 
euclidean space or euclidean half space) with linear $G$-action, where $M^{G}$ and $N^{G}$ denote the fixed point set of $M$ and $N$ respectively. We can choose the family $\left\{U_{i}\right\}$ such that, for any $k \in K$ and for any $i$, $f_{k}\left(\bar{U}_{i}\right)$ is contained in $V_{j}$ for some $j$ depending on $k$ and $i$. There exists a positive continuous function $\delta_{1}$ on $M$ as follows. For any continuous map $g: M \times K \rightarrow N$, such that $g_{k}$ is $\delta_{1}$-approximation to $f_{k}$ for each $k$, has these properties. Let $\left\{W_{i}\right\}$ be a family of invariant open sets of $M$ with $\bar{W}_{i} \subset U_{i}$ and $\cup \mathrm{W}_{i} \supset M^{G}$.

Let $C_{r}(y)$ denote a closed $r$-neighborhood of $y$ in $K$ for a positive number $r$ and $y \in K$. There exists a sufficiently small positive number $r$ such that, for any $z \in C_{r}(y)$ and for any $y \in K, f_{z}\left(\bar{U}_{1}\right)$ is contained in $V_{j}$ for some $j$ depending on $y$. Then we can find a finite number of $C_{r}(y)$, say $C_{n}=C_{r}\left(y_{n}\right)(n=1,2, \ldots, l)$, such that $\bigcup_{n=1}^{l} C_{n} \subset K-L^{\prime}$. We can assume that $C_{n} \cap L=\varnothing$ for any $n$. Let $\phi_{1}$ : $M \rightarrow I$ be an invariant smooth function on $M$ which equals 1 on $\bar{W}_{1}$ and 0 outside of $U_{1}$. Let $A_{n}$ and $B_{n}, n=1,2, \ldots, l$, be open sets of $K$ such that $\bar{A}_{n} \subset B_{n} \subset \bar{B}_{n} \subset$ int $C_{n}$ and $\bigcup_{n=1}^{l} A_{n}$ contains $K-L^{\prime}$. Let $\xi_{n}$ : $K \rightarrow I, n=1,2, \ldots, l$, be smooth functions on $K$ which equals 1 on $\bar{A}_{n}$ and 0 outside of $B_{n}$.

We shall identify $U_{i}$ and $V_{j}$ as euclidean spaces or euclidean half spaces with linear $G$-actions. For any $n$, we can find $j(n)$ such that $f_{z}\left(\bar{U}_{1}\right) \subset V_{j(n)}$ for any $z \in C_{n^{*}}$. Let $f^{1, n}: M \times C_{n} \rightarrow V_{j(n)}$ be a $G$-map defined by $f_{z}^{1, n}(x)=\phi_{1}(x) \cdot f_{z}(x)$ for $x \in U_{1}$ and $z \in C_{n}$, and $f^{1, n}=0$ outside of $U_{1} \times C_{n}$. Let $g^{1, n}: M \times K \rightarrow V_{j(n)}$ be a $G$-map defined by $g_{z}^{1, n}(x)=$ $\xi_{n}(z) \cdot f_{z}^{1, n}(x)$ for $x \in M$ and $z \in K$. Since $g_{z}^{1, n}=0$ for $z \notin C_{n}$, we can extend $g^{1, n}$ trivially on $M \times R$ (resp. $M \times R^{2}$ ) if $K=I$ (resp. $I \times I$ ). Define a smooth $G$-map $h^{1, n}: M \times K \rightarrow V_{j(n)}$ by

$$
h_{z}^{1, n}(x)=\int_{C\left(\varepsilon_{n}\right)} \varphi_{n}(y) \cdot g_{z+y}^{1, n}(x) d y \text { for } x \in M \text { and } z \in K,
$$

where $C\left(\varepsilon_{n}\right)$ is a closed $\varepsilon_{n}$-disc in $K$ and $\varphi_{n}$ is a smooth function on $R$ or $R^{2}$ which is positive on int $C\left(\varepsilon_{n}\right)$ and 0 outside of $C\left(\varepsilon_{n}\right)$ and $\int_{C\left(\varepsilon_{n}\right)} \varphi_{n}(y) d y=1$. Choose the positive number $\varepsilon_{n}$ less than the distance from $B_{n}$ to the complement of $C_{n}$. Then $h^{1, n}=0$ outside of $U_{1} \times C_{n}$.

Let $F^{1,0}=f$. Assume that $F^{1, n-1}: M \times K \rightarrow N$ is defined such that 
$F^{1, n-1}$ is smooth on $\bar{W}_{1} \times\left(\bar{A}_{1} \cup \ldots \cup \bar{A}_{n-1}\right)$ and $F^{1, n-1}=F^{1, n-2}$ outside of $U_{1} \times C_{n-1}$. Moreover we assume that $F_{k}^{1, n-1}$ is a $\delta_{1} / 2^{l-n+2}$-approximation to $F_{k}^{1, n-2}$ for each $k \in K$. Then $F_{z}^{1, n-1}$ is a $\delta_{1}$-approximation to $f_{z}$. Let $F^{1, n}: M \times K \rightarrow N$ be a $G$-map defined by

$$
F_{z}^{1, n}(x)=F_{z}^{1, n-1}(x)\left(1-\phi_{1}(x) \xi_{n}(z)\right)+h_{z}^{1, n}(x) \text {, for } x \in M \text { and } z \in K .
$$

Since $F^{1, n}=h^{1, n}$ on $\bar{W}_{1} \times \bar{A}_{n}, F^{1, n}$ is smooth on $\bar{W}_{1} \times\left(\bar{A}_{1} \cup \ldots \cup \bar{A}_{n}\right)$ (note that, if $F^{1, n-1}$ is smooth on a subset of $M \times K, F^{1, n}$ is smooth on the subset of $M \times K)$. Since $h^{1, n}=0$ outside of $U_{1} \times C_{n}, F^{1, n}=F^{1, n-1}$ outside of $U_{1} \times C_{n}$. By the argument of J. Munkres [4, Chapter I, $\S 4]$, we can choose the positive numbers $\varepsilon_{i}(i=1,2, \ldots, n)$ so small that $F_{k}^{1, n}$ is a $\delta_{1} / 2^{l-n+1}$-approximation to $F_{k}^{n-1}$ for each $k$. Then we can see that $F_{z}^{1, n}$ is a $\delta_{1}$-approximation to $f_{z}$ and $F_{z}^{1, n+1}$ is defined. By the induction, we have a $G$-map $F^{1, l}: M \times K \rightarrow N$ such that $F^{1, l}$ is smooth on $\bar{W}_{1} \times\left(\cup_{n=1}^{l} \bar{A}_{n}\right)$ and $F^{1, l}=F^{1, l-1}$ outside of $U_{1} \times C_{n}$. Set $F^{1}=F^{1, l}$. Since $\bigcup_{n=1}^{l} \bar{A}_{n}$ contains $K-L^{\prime}, F^{1}$ is smooth on $\bar{W}_{1} \times K$. And since $C_{n} \cap L={ }_{n=1}=\sigma^{1}, F^{1}=f$ on $M \times L$.

There exists a positive continuous function $\delta \leqq \delta_{1}$ on $M$ such that, for each $k \in K$, any $C^{1}$-map from $M$ to $N$, which is a $\delta$-approximation in $C^{1}$-topology to $f_{k}$, is an imbedding (see J. Munkres [4, Chapter I, Theorem 3.10]). We can choose the positive numbers $\varepsilon_{n}, n=$ $1,2, \ldots, l$, so small that $F_{k}^{1}$ is a $\delta / 2$-approximation to $f_{k}$ in $C^{1}$-topology for each $k \in K$.

By the induction we have $G$-maps $F^{i}: M \times K \rightarrow N(i=2,3, \ldots)$, which is smooth on $\left(\bar{W}_{1} \cup \ldots \cup \bar{W}_{i}\right) \times K$, such that $F^{i}=f$ on $M \times L$ and $F^{i}=F^{i-1}$ outside of $U_{i} \times K$. Moreover we can choose $F_{k}^{i}$ is a $\delta / 2^{i}$-approximation to $F_{k}^{i-1}$ in $C^{1}$-topology for each $k \in K$. Define a $G$-map $F: M \times K \rightarrow N$ by $F_{k}(x)=\lim _{i \rightarrow \infty} F_{k}^{i}(x) ; F_{k}$ is well defined because $F_{k}^{i}=F_{k}^{i+1}=\ldots$ on some neighborhood of $x$, for sufficiently large i. $\quad F: M \times K \rightarrow N$ is smooth on $\left(\cup_{i} \bar{W}_{i}\right) \times K$ and $F=f$ on $M \times$ L. Moreover $F_{k}$ is a $\delta$-approximation to $f_{k}$ in $C^{1}$-topology, for each $k \in K$.

Let $T$ be a closed invariant neighborhood of $M^{G}$ in $M$ such that $T$ is contained in $\bigcup_{i} W_{i} \cdot \quad F_{k}\left(M-M^{G}\right)$ is contained in $N-N^{G}$, for 
each $k$, since $F_{k}$ is a $G$-imbedding. Let $\bar{F}:\left(M-M^{G}\right) / G \times K \rightarrow(N-$ $\left.N^{G}\right) / G$ be the orbit map of $F$. Then $\bar{F}$ is a smooth map on a neighborhood of $\left(T-M^{G}\right) / G \times K$ and $\bar{F}=\bar{f}$ on $\left(M-M^{G}\right) / G \times L$, and $\bar{F}_{k}$ is a $\delta$-approximation to $\bar{f}_{k}$ for each $k \in K$, where $\bar{f}_{k}$ is the orbit map of $f_{k}$. By the relative version of the argument of $\mathrm{J}$. Munkres [4, Chapter I, §4], we have a smooth map $\bar{H}:\left(M-M^{G}\right) / G \times K \rightarrow$ $\left(N-N^{G}\right) / G$ such that $\bar{H}=\bar{F}$ on $\left(T-M^{G}\right) / G \times K$ and $\bar{H}$ is homotopic to $\bar{F}$ relative to $\left(T-M^{G}\right) / G \times K \cup\left(M-M^{G}\right) / G \times L$. Moreover $\bar{F}_{k}$ is a $\delta$-approximation to $\bar{f}_{k}$ in $C^{1}$-topology, for each $k \in K$. By the covering homotopy property, we have a smooth $G$-map $H$ : ( $M-$ $\left.M^{G}\right) \times K \rightarrow N-N^{G}$ whose orbit map is $\bar{H}$. Define $H=F$ on $T \times K$. Then $H: M \times K \rightarrow N$ is a smooth $G$-map such that $H_{k}$ is a $\delta$-approximation to $f_{k}$ in $C^{1}$-topology, for each $k \in K$, and $H=f$ on $M \times L$. This completes the proof of Lemma 1.2.

Let $f: S^{\mathrm{V}} \rightarrow S^{\mathrm{W}}$ be a $G$-imbedding. The fixed point set of $S^{\mathrm{v}}$ and $S^{W}$ are $S^{n}$ and $S^{d n+k}$ respectively. Let $f^{G}: S^{n} \rightarrow S^{d n+k} \subset S^{W}$ denote an imbedding which is a restriction of $f$ to $S^{n}$. Let $j: S^{\mathrm{v}} \rightarrow S^{\mathrm{W}}$ be the standard imbedding.

Proposition 1. 3. Let $f_{0}: S^{\mathrm{V}} \rightarrow S^{\mathrm{W}}$ be a $G$-imbedding. Then there exists a G-isotopy $f: S^{v} \times I \rightarrow S^{\mathrm{W}}$ between $f_{0}$ and $f_{1}$ with $f_{1}^{G}=j$ on $S^{n}$.

Proof. Since $d n+k>2 n$, we have an isotopy $h: S^{d n+k} \times I \rightarrow S^{d_{n+k}}$ such that $h_{0}=1$ and $h_{1} \cdot f_{0}^{G}=j$. By the isotopy extension theorem, there exists an isotopy $H: S^{\mathrm{w}} \times I \rightarrow S^{\mathrm{W}}$ such that $H_{0}=1$ and $H=h$ on $S^{a_{n+k}} \times I$. Using a result of G. Bredon [1, Chapter VI, Theorem 3.1], we have a $G$-isotopy $K: S^{\mathrm{W}} \times I \rightarrow S^{\mathrm{W}}$ such that $K_{0}=1$ and $K=H$ on $H^{G} \times I$, where $H^{G}=\left\{x \in S^{W} ; H_{t}(g \cdot x)=g \cdot H_{t}(x)\right.$ for any $t \in \mathrm{I}$ and $g \in G\}$. Note that $S^{a n+k} \subset H^{G}$. Let $f: S^{v} \times I \rightarrow S^{W}$ be a $G$-isotopy between $f_{0}$ and $f_{1}$ defined by $f_{t}=K_{t} \cdot f_{0}$. Then $f_{1}^{G}=j$ and this completes the proof of Proposition 1.3.

Proposition 1. 4. Let $f: S^{\mathrm{v}} \times I \rightarrow S^{\mathrm{W}}$ be a G-isotopy with $f_{i}^{G}=j$ for $i=0,1$. Then there exists a G-isotopy $h: S^{\mathrm{v}} \times I \rightarrow S^{\mathrm{w}}$ such that 
$h_{i}=f_{i}$ for $i=0,1$ and $h_{i}^{G}=j$ for $0 \leqq t \leqq 1$.

Proof. Let $f: S^{\mathrm{v}} \times I \rightarrow S^{\mathrm{w}} \times I$ be a $G$-imbedding defined by $f(x, t)$ $=\left(f_{t}(x), t\right)$. Let $f^{G}: S^{n} \times I \rightarrow S^{d_{n+k}} \times I$ be an imbedding which is a restriction of $f$ to $S^{n} \times I$. Let $E\left(S^{n}, S^{a_{n}+k}\right)$ denote the set of all imbeddings of $S^{n}$ into $S^{a_{n+k}}$ with $C^{\infty}$-topology. By a result of J. Dax [2, Chapter VI, §3], $\pi_{1}\left(E\left(S^{n}, S^{d n+k}\right)\right)=0$ since $d n+k>2 n+2$. Then we have a continuous map $a: I \times I \rightarrow E\left(S^{n}, S^{d_{n+k}}\right)$ such that, for a sufficiently small $\varepsilon>0$,

$$
a(t, s)=\left\{\begin{array}{l}
f_{t}^{G} \text { for }(t, s) \in I \times[0, \varepsilon] \\
j \text { for }(t, s) \in[0, \varepsilon] \times I \cup I \times[1-\varepsilon, 1] \cup[1-\varepsilon, 1] \times \mathrm{I} .
\end{array}\right.
$$

Using Lemma 1. 2, we may assume that $\hat{a}: S^{n} \times I \times I \rightarrow S^{d_{n+k}} \times I$ is an 1sotopy, where $\hat{a}(x, t, s)=(a(t, s)(x), t)$. Then we have an imbedding $\tilde{a}: S^{n} \times I \times R \rightarrow S^{a+k} \times I \times R$ defined by

$$
\tilde{a}(x, t, s)= \begin{cases}\hat{a}(x, t, s), s) & \text { for } 0 \leqq s \leqq 1 \\ \hat{a}(x, t, 0), s) & \text { for } s<0 \\ \hat{a}(x, t, 1), s) & \text { for } s>1 .\end{cases}
$$

$\tilde{a}\left(S^{n} \times I \times R\right)$ is a closed $G$-submanifold of $S^{\mathbb{W}} \times I \times R$, and $\tilde{a}\left(S^{n} \times I \times R\right)$ intersects normally on $\partial\left(S^{n} \times I \times R\right)$ with respect to a product $G$-invariant Riemannian metric on $S^{\mathrm{w}} \times I \times R$. By using the proof of G. Bredon [1, Chapter IV, Theorem 2.2] with respect to the Riemannian metric, we have an invariant open $\delta$-tubular neighborhood $N$ of $\tilde{a}\left(S^{n} \times I \times R\right)$, where $\delta$ is a $G$-invariant positive real valued function on $\tilde{a}\left(S^{n} \times I \times R\right)$.

The tangent vectors to the curves $\tilde{a}(x \times t \times R)$ define an invariant vector field $\tilde{X}$ on $\tilde{a}\left(S^{n} \times I \times R\right)$ of the form $\tilde{X}(\tilde{a}(x, t, s))=(X(x, s$, $t), \quad 0,1) \in T_{a(x, s, t)}\left(S^{\mathrm{w}} \times I \times R\right)$, where $T\left(S^{\mathrm{w}} \times I \times R\right)$ is the tangent bundle of $S^{\mathrm{w}} \times I \times R$. Identifying $N$ with a $G$-invariant normal bundle to $\tilde{a}\left(S^{n} \times I \times R\right)$ in $S^{\mathrm{w}} \times I \times R$, we denote $p: N \rightarrow \tilde{a}\left(S^{n} \times I \times R\right)$ the bundle projection. Let $r: I \rightarrow R$ be a $C^{\infty}$-function such that $r(t)=$ 1 for $0 \leqq t \leqq 1 / 3,0<r(t)<1$ for $1 / 3<t<2 / 3$ and $r(t)=0$ for $2 / 3 \leqq$ $t \leqq 1$. Let $Y$ be a $G$-invariant vector field on $S^{\mathrm{w}} \times I \times R$ defined by $Y(v)=r(\|v\| / \delta(p(v))) \cdot X(p(v))$ for $v \in N$ and $Y=0$ on the outside of $N$, where \|\| denote the $G$-invariant metric of $S^{w} \times I \times R$. 
Since $\tilde{a}(x, t, s)=(j(x), t, s)$ for $0 \leqq t \leqq \varepsilon$ and $1-\varepsilon \leqq t \leqq 1$, and since $\tilde{a}(x, t, s)=(\hat{a}(x, t, 0), s)$ for $s \leqq 0$ and $\tilde{a}(x, t, s)=(\hat{a}(x, t, 1), s)$ for $s \geqq 1, \operatorname{Supp}(Y)$ is contained in $S^{\mathrm{W}} \times[\varepsilon, 1-\varepsilon] \times I$ which is compact. We can regard $Y$ as a time-dependent $G$-invariant vector field on $S^{W} \times I$, and $Y$ generates a $G$-isotopy $F: S^{\mathrm{w}} \times I \times I \rightarrow S^{\mathrm{w}} \times I$ (see $\mathrm{M}$. Hirsch [3, Chapter 8, Theorem 1.1]). Since $I$ component of $Y$ is 0 , each $F_{s}: S^{\mathrm{W}} \times I \rightarrow S^{\mathrm{W}} \times I$ is level preserving. Let $h: S^{\mathrm{v}} \times I \rightarrow S^{\mathrm{W}}$ be a $G$-isotopy defined by $h=p_{1} \cdot F_{1} \cdot \tilde{f}$, where $p_{1}: S^{\mathrm{W}} \times I \rightarrow S^{\mathrm{W}}$ is the projection on the first factor. Then $h_{t}=f_{t}$ for $t=0,1$ and $h_{t}=j$ on $S^{n}$ for each $t$. This completes the proof of Proposition 1.4.

\section{§ 2. Linearlity on a Neighborhood of the Fixed Point Set}

In this section we shall prove that any $G$-imbedding of $S^{\mathrm{V}}$ into $S^{\text {w }}$ is $G$-isotopic to a $G$-imbedding which is linear on a neighborhood of $S^{n}$ (see Proposition 2.1), and if two $G$-imbeddings of $S^{v}$ into $S^{w}$, which are linear on a neighborhood of $S^{n}$, are $G$-isotopic, then there exists a $G$-isotopy between them which is linear on a neighborhood of $S^{n}$ (see Proposition 2.3).

Since the fixed point set of $S^{v}$ is $S^{n}$ and since $S^{v}$ is a $G$-submanifold of $S^{\mathrm{w}}$, we can regard $S^{n}$ as a $G$-submanifold of $S^{\mathrm{w}}$. Let $U$ and $N$ denote invariant open tubular neighborhoods of $S^{n}$ in $S^{v}$ and $S^{n}$ in $S^{\mathrm{W}}$ respectively. We shall identify $U$ and $N$ with invariant normal bundles to $S^{n}$ in $S^{v}$ and to $S^{n}$ in $S^{\mathrm{W}}$ respectively. Let $f: S^{v}$ $\rightarrow S^{\text {w }}$ be a $G$-imbedding with $f^{G}=j$. We shall assume that $f(U)$ is contained in $N$. Let $f^{\prime}: U \rightarrow N$ be a $G$-bundle monomorphism defined by the differential of $f$.

Proposition 2.1. Let $f: S^{\mathrm{v}} \rightarrow S^{\mathrm{w}}$ be a G-imbedding with $f^{G}=j$. Then there exists a G-isotopy $h: S^{\mathrm{v}} \times I \rightarrow S^{\mathrm{W}}$ such that $h_{0}=f$ and $h_{1}=$ $f^{\prime}$ on some invariant neighborhood of $S^{n}$ in $S^{v}$.

In order to prove Proposition 2.1, we start with the following lemma. 
Lemma 2.2. Let $M$ be a G-submanifold of a G-manifold $N$. Let $f: M \times I \rightarrow N$ be a $G$-isotopy such that $f_{t}(\partial M) \subset \partial N$ and $f_{t}(M)$ intersects transversally on $\partial N$ for each $t$. Let $A$ be an invariant subspace of $M$ such that $\bar{A}$ is compact. Then there exists a G-isotopy $F: N \times I \rightarrow N$ such that $F_{0}=1$ and $F_{t} \cdot f_{0}=f_{t}$ on $A$ for $0 \leqq t \leqq 1$.

Proof. Let $\tilde{f}: M \times R \rightarrow N \times R$ be a $G$-imbedding defined by

$$
\tilde{f}(x, t)=\left\{\begin{array}{l}
\left(f_{t}(x), t\right) \text { for } 0 \leqq t \leqq 1 \\
\left(f_{0}(x), t\right) \text { for } \mathrm{t}<0 \\
\left(f_{1}(x), t\right) \text { for } t>1 .
\end{array}\right.
$$

We can assume that $G$ acts by isometries in some product metric on $N \times R$. Let $\nu$ be an invariant normal bundle of $\tilde{f}(M \times R)$ in $N \times R$ and let $p: \nu \rightarrow \tilde{f}(M \times R)$ be the projection. Then the exponential map is defined on some neighborhood of $\tilde{f}(M \times R)$ in $\nu$ and is an equivariant immersion on a smaller invariant open neighborhood of $\tilde{f}(M \times R)$ (see the proof of G. Bredon [1, Chapter VI, Theorem 2. 2]). Let $B$ be an invariant open neighborhood of $\bar{A}$ such that $\bar{B}$ is compact. Since $\bar{B}$ is compact, the exponential map is a $G$-imbedding on an invariant neighborhood of $\tilde{f}(\bar{B} \times I)$ in $\nu \mid \tilde{f}(\bar{B} \times I)$. By a method of the proof of G. Bredon [1, Chapter VI, Theorem 2. 2], we have a $G$-imbedding $\varphi: \nu \mid \tilde{f}(\bar{B} \times I) \rightarrow N \times R$. We shall identify $\nu \mid \tilde{f}(\bar{B} \times I)$ as the image of $\varphi$.

The tangent vectors to the curves $\tilde{f}(x \times R) \quad(x \in M)$ define an invariant vector field $\tilde{X}$ on $\tilde{f}(M \times R)$ of the form $\tilde{X}(\tilde{f}(x, t))=(X(x$, $t), 1) \in T_{f(x, t)}(N \times R)$. Note that $\operatorname{Supp}(X)$ is contained in $\tilde{f}(M \times I)$. Take an invariant $C^{\infty}$-partition of unity subordinate to the covering $\{B, M-\bar{A}\}$ of $M$, and let $u$ be the invariant function correspondence to $B$. Let $X^{\prime}$ be an invariant vector fields on $\tilde{f}(M \times R)$ defined by $X^{\prime}(\tilde{f}(x, t))=u(x) \cdot X(x, t)$ and $X^{\prime}=0$ outside of $\tilde{f}(B \times R)$. Then $\operatorname{Supp}\left(X^{\prime}\right)$ is contained in $\tilde{f}(\bar{B} \times I)$ and $X^{\prime}=X$ on $\tilde{f}(\bar{A} \times R)$.

Let $r: R \rightarrow[0,1]$ be a $C^{\infty}$-function such that $r(t)=1$ for $t \leqq 1$, $0<r(t)<1$ for $1<t<2$ and $r(t)=0$ for $t \geqq 2$. Let $Y$ be an invariant vector field on $N \times I$ defined by $Y(v)=r(\|v\|) \cdot X^{\prime}(p(v))$ on $\nu \mid \tilde{f}(\bar{B} \times$ $I)$ and $Y=0$ outside of $\nu \mid \tilde{f}(\bar{B} \times I)$, where $\|$ i| is an invariant Rie- 
mannian metric on $\nu$. Then we can regard $Y$ as a time-dependent invariant vector field on $N$. Note that $\operatorname{Supp}(Y)$ is contained in $\nu(2)$ $\mid \bar{B} \times I$ which is compact, where $\nu(2)=\{v \in \nu ;\|v\| \leqq 2\}$. Therefore $Y$ generates a $G$-isotopy $F: N \times I \rightarrow N$ such that $F_{0}=1$ and $F_{t} \cdot f_{0}=f_{t}$ on $A$ for $0 \leqq t \leqq 1$. This completes the proof of Lemma 2. 2 .

Proof of Proposition 2. 1. Let $g: U \times I \rightarrow N \hookrightarrow S^{W}$ be a homotopy of $G$-imbeddings defined by $g_{t}(v)=1 /(1-t) \cdot f((1-t) v)$ for $0 \leqq t<1$ and $v \in U$, and $g_{1}=f^{\prime}$. Note that $g_{0}=f \mid U, \lim _{t \rightarrow 1} g_{t}=f^{\prime}$ and $g_{t}$ is a $G$-imbedding for each $t$. By Lemma 1.2 we can assume that $g$ is a $G$-isotopy between $f \mid U$ and $f^{\prime}$. By Lemma 2.2 there exists a $G$-isotopy $G: S^{\mathrm{W}} \times I \rightarrow S^{\mathrm{W}}$ such that $F_{0}=1$ and $F_{t} \cdot g_{0}=g_{t}$ on some neighborhood of $S^{n}$. Let $h: S^{v} \times I \rightarrow S^{w}$ be a $G$-isotopy defined by $h_{t}=F_{t} \cdot f$. Then $h_{0}=f$ and $h_{1}=f^{\prime}$ on some neighborhood of $S^{n}$. This completes the proof of Proposition 2. 1 .

By Proposition 1.3 and Proposition 2. 1, any element of $I\left(S^{v}, S^{W}\right)$ is represented by a $G$-imbedding $f: S^{v} \rightarrow S^{W}$ such that $f^{G}=j$ and $f=f^{\prime}$ on an invariant tubular neighborhood of $S^{n}$.

Proposition 2.3. Let $f: S^{v} \times I \rightarrow S^{W}$ be a G-isotopy such that $f_{t}^{G}=j(0 \leqq t \leqq 1)$ and $f_{i}=f_{i}^{\prime}(i=0,1)$ on an invariant tubular neighborhood $U$ of $S^{n}$. Then there exists a G-isotopy $h: S^{v} \times I \rightarrow S^{w}$ such that $h_{i}=f_{i}(i=0,1)$ and $h_{t}=h_{t}^{\prime}$ on an invariant neighborhood of $S^{n}$ for $0 \leqq t \leqq 1$.

Proof. Let $\tilde{f}: S^{v} \times I \rightarrow S^{\mathrm{w}} \times I$ be a $G$-imbedding defined by $\tilde{f}(x, t)=$ $\left(f_{t}(x), t\right)$. We can assume that $f_{t}(U)$ is contained in $N$ for each $t$. Let $\tilde{f}^{\prime}: U \times I \rightarrow N \times I$ be a $G$-imbedding defined by $\tilde{f}^{\prime}(v, t)=\left(f_{t}^{\prime}(v)\right.$, t). Let $F: U \times I \times I \rightarrow N \times I$ be a $G$-map defined by $F_{s}(v, t)=(1 /(1-$ $\left.s) \cdot f_{t}((1-s) v), t\right)$ for $0 \leqq s<1$ and $F_{1}=f^{\prime}$. Then $F_{0}=\tilde{f}$ and $\lim _{s \rightarrow 1}$ $F_{s}=\tilde{f}^{\prime}$ and $F_{s}$ is a $G$-imbedding for each $s$. Note that, by the definition of $G$-isotopy, there exists a positive number $\varepsilon$ such that $f_{t}=f_{0}$ for $0 \leqq t \leqq \varepsilon$ and $f_{t}=f_{1}$ for $1-\varepsilon \leqq t \leqq 1$. Thus $F_{s}=f_{0}^{\prime} \times 1$ for $0 \leqq t \leqq \varepsilon$ and $F_{s}=f_{1}^{\prime} \times 1$ for $1-\varepsilon \leqq t \leqq 1$. By Lemma 1.2 we can assume that $F$ 
is a $G$-isotopy between $\tilde{f} \mid U \times I$ and $\tilde{f}^{\prime}$. Let $\tilde{F}: U \times I \times R \rightarrow N \times I \times$ $R \hookrightarrow S^{W} \times I \times R$ be a $G$-imbedding defined by

$$
\tilde{F}(x, t, s)=\left\{\begin{array}{l}
(F(x, t, s), s) \text { for } 0 \leqq s \leqq 1 \\
(F(x, t, 0), s) \text { for } s<0 \\
(F(x, t, 1), s) \text { for } s>1 .
\end{array}\right.
$$

Let $U$ and $U_{2}$ be invariant open tubular neighborhoods of $S^{n}$ such that $\bar{U}_{1} \subset U_{2} \subset \bar{U}_{2} \subset U$. Let $\nu$ be an invariant normal bundle of $\widetilde{F}(U \times I \times R)$ in $S^{\mathrm{w}} \times I \times R$ and let $p: \nu \rightarrow \widetilde{F}(U \times I \times R)$ be the projection. Similarly as the proof of Lemma 2.2, we have a $G$-imbedding $\varphi: \nu \mid \widetilde{F}\left(\bar{U}_{2} \times I \times I\right) \rightarrow S^{w} \times I \times R$. We shall identify $\nu \mid \widetilde{F}\left(\bar{U}_{2} \times I \times I\right)$ as the image of $\varphi$.

The tangent vectors to the curves $\widetilde{F}(x \times t \times R)(x \times t \in U \times I)$ define an invariant vector field $\tilde{X}$ on $\tilde{F}(U \times I \times R)$ of the form $\tilde{X}(\tilde{F}(x, t, s))=$ $(X(x, t, s), 0,1) \in T_{\tilde{F}(x, t, s)}(N \times I \times R)$. Note that $\operatorname{Supp}(X)$ is contained in $\tilde{F}(U \times[\varepsilon, 1-\varepsilon] \times I)$. Take an invariant partition of unity subordinate to the covering $\left\{U_{2}, U-\bar{U}_{1}\right\}$ of $U$, and $u$ be the invariant $C^{\infty}$-function corresponding to $U_{2}$. Let $X^{\prime}$ be an invariant vector field on $\tilde{F}(U \times I \times R)$ defined by $X^{\prime}(\tilde{F}(x, t, s))=u(x) \cdot X(x$, $t, s)$ for $(x, t, s) \in \bar{U}_{2} \times I \times R$ and $X^{\prime}=0$ outside of $\tilde{F}\left(\bar{U}_{2} \times I \times R\right)$. Then $\operatorname{Supp}\left(X^{\prime}\right)$ is contained in $\widetilde{F}\left(\bar{U}_{2} \times[\varepsilon, 1-\varepsilon] \times I\right)$ and $X^{\prime}=X$ on $\tilde{F}\left(\bar{U}_{1} \times I \times R\right)$.

Let $r: R \rightarrow[0,1]$ be a $C^{\infty}$-function such that $r(t)=1$ for $t \leqq 1$, $0<r(t)<1$ for $1<t<2$ and $r(t)=0$ for $t \geqq 2$. Let $Y$ be an invariant vector field on $S^{\mathrm{W}} \times I \times R$ defined by $Y(v)=r(\mid i v \|) \cdot X^{\prime}(p(v))$ for $v \in$ $\nu \mid \widetilde{F}\left(\bar{U}_{2} \times I \times I\right)$ and $Y=0$ outside of $\nu \mid F\left(\bar{U}_{2} \times I \times I\right)$, where \|\| is an invariant Riemannian metric on $\nu$. Then we can regard $Y$ as a time-dependent invariant vector field on $S^{\mathrm{w}} \times I$. Note that $\operatorname{Supp}(Y)$ is contained in $\nu(2) \mid \widetilde{F}\left(\bar{U}_{1} \times[\varepsilon, 1-\varepsilon] \times I\right)$, where $\nu(2)=\{v \in \nu:\|v\| \leqq$ 2\}. Then $Y$ generates a $G$-isotopy $H: S^{w} \times I \times I \rightarrow S^{W} \times I$ such that $H_{0}=1$ and $H_{s} \cdot F_{0}=F_{s}$ on $\bar{U}_{1} \times I$ for $0 \leqq s \leqq 1$. Since $I$ component of $Y$ is 0 , each $H_{s}: S^{w} \times I \rightarrow S^{w} \times I$ is level preserving equivariant diffeomorphism. Let $h: S^{v} \times I \rightarrow S^{w}$ be a $G$-isotopy defined by $h=p_{1} \cdot H_{1} \cdot \tilde{f}$, where $p_{1}: S^{\mathrm{W}} \times I \rightarrow S^{\mathrm{W}}$ is the projection on the first factor. Then $h_{i}=f_{\mathrm{t}}$ for $i=0,1$ and $h_{t}=h_{t}^{\prime}$ on $U_{1}$. This completes the proof of 
Proposition 2.3.

Definition 2. 4. Let $f_{i}: S^{v} \rightarrow S^{W} \quad(i=0,1)$ be $G$-imbeddings such that $f_{i}=f_{i}^{\prime}$ on $U$. $f_{0}$ and $f_{1}$ are said to be equivalent if there exists a $G$-isotopy $f: S^{v} \times I \rightarrow S^{W}$ between $f_{0}$ and $f_{1}$ such that $f_{t}=f_{t}^{\prime}$ on some neighborhood of $S^{n}$. Let $I_{1}\left(S^{v}, S^{\mathrm{w}}\right)$ denote the set of all equivalence classes of these $G$-imbeddings.

Corollary 2.5. The natural map $i_{1}: I_{1}\left(S^{v}, S^{w}\right) \rightarrow\left(S^{v}, S^{w}\right)$ is bijective.

Proof. By Proposition 1.3 and Proposition 2.2, $i_{1}$ is surjective. By Proposition 1.4 and Proposition 2.3, $i_{1}$ is injective, and Corollary 2.5 follows.

\section{§ 3. Orthogonality on a Neighborhood of the Fixed Point Set}

In this section we shall prove that any $G$-imbedding from $S^{v}$ into $S^{W}$ is $G$-isotopic to a $G$-imbedding which is orthogonal on a neighborhood of $S^{n}$. Moreover we shall prove that, if two $G$-imbeddings $f_{0}$ and $f_{1}$, which are orthogonal on $U$, coincide on $U$, then there exists a $G$-isotopy $f$ between $f_{0}$ and $f_{1}$ such that $f_{t}=f_{0} \quad(0 \leqq t \leqq 1)$ on $U_{1}$, where $U$ and $U_{1}$ are invariant neighborhood of $S^{n}$.

As in $\S 2$, let $U$ and $N$ be invariant normal bundles of $S^{n}$ in $S^{v}$ and to $S^{n}$ in $S^{W}$ respectively. Note that $U$ and $N$ are isomorphic to product bundles $S^{n} \times V_{1}$ and $S^{n} \times\left(d V_{1} \oplus R^{(d-1) n+k}\right)$ as a $G$-vector bundles over $S^{n}$ respectively. Let $f: S^{v} \rightarrow S^{W}$ be a $G$-imbedding with $f^{G}=j$. Then $f^{\prime}: U \rightarrow N$ induces a continuous map

$$
\hat{f}: S^{n} \rightarrow \operatorname{Mon}^{G}\left(V_{1}, d V_{1} \oplus R^{(d-1) n+k}\right),
$$

where $\operatorname{Mon}^{G}\left(V_{1}, d V_{1} \oplus R^{(d-1) n+k}\right)$ is the set of all $G$-module monomorphisms from $V_{1}$ to $d V_{1} \oplus R^{(d-1) n+k}$ with usual topology. By Schur's lemma, $\operatorname{Mon}^{G}\left(V_{1}, d V_{1} \oplus R^{(d-1) n+k}\right)$ is isomorphic to $\operatorname{Mon}^{G}\left(V_{1}, d V_{1}\right)$. 
Proposition 3.1. Let $f: S^{V} \rightarrow S^{\mathrm{W}}$ be a G-imbedding with $f^{G}=j$. Let $h: S^{n} \times I \rightarrow \operatorname{Mon}^{G}\left(V_{1}, d V_{1}\right)$ be a homotopy with $h_{0}=\hat{f}$. Then there exists a G-isotopy $F: S^{v} \times I \rightarrow S^{W}$ such that $F_{0}=f$ and $\hat{F}_{1}=h_{1}$.

Proof. Let $p: U \rightarrow S^{n}$ be the bundle projection. Let $F^{\prime}: U \times I \rightarrow N$ be a homotopy of $G$-imbeddings defined by $F_{t}^{\prime}(u)=h_{t}(p(u))(u)$ for $u \in U$. Then, by Lemma 1.2, we can assume that $F^{\prime}$ is a $G$-isotopy. By Lemma 2.2, we have a $G$-isotopy $H: S^{\mathrm{W}} \times I \rightarrow S^{\mathrm{W}}$ such that $H_{0}=1$ and $H_{t} \cdot f^{\prime}=F_{t}^{\prime}$ on some invariant neighborhood of $S^{n}$ for each $t$. Let $F: S^{v} \times I \rightarrow S^{\text {w }}$ be a $G$-isotopy defined by $F_{t}=H_{t} \cdot f$. Then $F_{0}=f$ and $\hat{F}_{1}=h_{1}$, and this completes the proof of Proposition 3.1.

Let $O^{G}\left(V_{1}, d V_{1}\right)$ denote the set of all $G$-module orthogonal monomorphisms from $V_{1}$ to $d V_{1}$. Let $F$ denote the field of real numbers $R$, complex numbers $C$ or quaternionic numbers $H$. Let $U(q, F)$ denote the orthogonal group $O(n)$, the unitary group $U(n)$ or the symplectic group $S p(n)$ in the case of $F=R, C$ or $H$ respectively. Let $\operatorname{Hom}^{G}\left(V_{1}, V_{1}\right)$ denote the group of $G$-module endmorphisms of $V_{1}$. Let $V_{r, s}^{\prime}(F)$ denote the Stiefel manifold (over $F$ ) of $s$-frames in $F^{r}$.

Lemma 3. 2. Suppose that $V_{1}$ is isomorphic to $\oplus_{i} k_{i} W_{i}$, where $W_{i}$ runs over the inequivalent irreducible real G-modules. Then

$$
\operatorname{Mon}^{G}\left(V_{1}, d V_{1}\right)=\prod_{i} V_{d k_{i} \cdot k_{i}}^{\prime}\left(F_{i}\right)
$$

and

$$
O^{G}\left(V_{1}, d V_{1}\right)=\prod_{i} U\left(d k_{i}, F_{i}\right) / U\left((d-1) k_{i}, F_{i}\right),
$$

where $F_{i}=R, C$ and $H$ when $\operatorname{dim} \operatorname{Hom}^{G}\left(W_{i}, W_{i}\right)=1,2$ and 4 respectively.

Proof. If $W_{i}$ is a real restriction of an irreducible complex (resp. quaternionic) $G$-module $W_{i}^{\prime}$, then $\operatorname{Hom}^{G}\left(W_{i}, W_{i}\right)$ is isomorphic to $C$ (resp. $H$ ) given by the scalar multiplication of $W_{i}^{\prime}$. Otherwise $\operatorname{Hom}^{G}\left(W_{i}, W_{i}\right)$ is isomorphic to $R$ given by the scalar multiplication of $W_{i}$ (see J. -P. Serre $\left.[6,13.2]\right)$. Therefore $\operatorname{Mon}^{G}\left(k_{i} W_{i}, d k_{i} W_{i}\right)$ 
and $O^{G}\left(k_{i} W_{i}, d k_{i} W_{i}\right)$ are identified with $V_{d k_{i}, k_{i}}^{\prime}\left(F_{i}\right)$ and $U\left(d k_{i}, F_{i}\right) /$ $U\left((d-1) k_{i}, F_{i}\right)$ respectively. By Schur's lemma $\operatorname{Hom}^{G}\left(V_{1}, d V_{1}\right)$ is isomorphic to $\oplus \operatorname{Hom}^{G}\left(k_{i} W_{i}, d k_{i} W_{i}\right)$. Then $\operatorname{Mon}^{G}\left(V_{1}, d V_{1}\right)$ and $O^{G}\left(V_{1}, d V_{1}\right)$ are identified with $\prod_{i} \operatorname{Mon}^{G}\left(k_{i} W_{i}, d k_{i} W_{i}\right)$ and $\prod_{i} O^{G}$ $\left(k_{i} W_{i}, d k_{i} W_{i}\right)$ respectively. This completes the proof of Lemma 3.2.

Proposition 3. 3. Let $f: S^{\mathrm{v}} \times I \rightarrow S^{\mathrm{w}}$ be a $G$-isotopy such that $f_{t}^{G}=j, f_{t}=f_{t}^{\prime}$ on $U$ for each $t$ and $\hat{f}_{0}=\hat{f}_{1}$. If $\pi_{n+1}\left(\operatorname{Mon}^{G}\left(V_{1}, d V_{1}\right)\right)=0$, then there exists a G-isotopy $h: S^{\mathrm{V}} \times I \rightarrow S^{\mathrm{W}}$ such that $h_{i}=f_{i}$ for $i=0,1$ and $\hat{h}_{t}=\hat{f}_{0}$ for $0 \leqq t \leqq 1$.

Proof. Let $a_{f}: S^{n} \times \partial(I \times I) \rightarrow \operatorname{Mon}^{G}\left(V_{1}, V_{1}\right)$ be a continuous map defined by

$$
a_{f}(x, t, s)=\left\{\begin{array}{l}
\hat{f}_{t}(x) \text { for } s=0 \text { and } 0 \leqq t \leqq 1 \\
\hat{f}_{0}(x) \text { for } s=1 \text { and } 0 \leqq t \leqq 1 \\
\hat{f}_{0}(x) \text { for } t=0,1 \text { and } 0 \leqq s \leqq 1 .
\end{array}\right.
$$

Since $\pi_{n+1}\left(\operatorname{Mon}^{G}\left(V_{1}, d V_{1}\right)\right)=0$, the only obstruction to extend $a_{f}$ to $S^{n} \times I \times I$ is a well defined cohomology class $o\left(a_{f}\right) \in H^{2}\left(S^{n} \times I \times I, S^{n} \times\right.$ $\partial(I \times I) ; \pi_{1}\left(\operatorname{Mon}^{G}\left(V_{1}, d V_{1}\right)\right)=\pi_{1}\left(\operatorname{Mon}^{G}\left(V_{1}, d V_{1}\right)\right)$. If $d \geqq 3, \operatorname{Mon}^{G}$ $\left(V_{1}, d V_{1}\right)$ is 2-connected by Lemma 3.2, and $o\left(a_{f}\right)=0$.

Now we will consider the case of $d=1$. In this case $\operatorname{Mon}^{G}\left(V_{1}, d V_{1}\right)$ is a group $A^{G}\left(V_{1}\right)$, where $A^{G}\left(V_{1}\right)$ is the group of all $G$-module automorphisms of $V_{1}$. Let $b_{f}: \partial(I \times I) \rightarrow \operatorname{Mon}^{G}\left(V_{1}, d V_{1}\right)=A^{G}\left(V_{1}\right)$ be a continuous map defined by $b_{f}(x)=a_{f}(*, x)$ for $x \in \partial(I \times I)$, where * is a point of $S^{n}$. Then the above obstruction class $o\left(a_{f}\right)$ is represented by $b_{f}$. Note that an element of $A^{G}\left(V_{1}\right)$ can be regarded as an equivariant linear diffeomorphism of $S^{\mathrm{W}}$ in the natural way. Let $g: S^{\mathrm{v}} \times I \rightarrow S^{\mathrm{W}}$ be a $G$-isotopy between $f_{0}$ and $f_{1}$ defined by $g_{t}=\hat{f}_{0}(*) \cdot \hat{f}_{t}(*)^{-1} \cdot f_{t}$. Then $b_{g}(x)=\hat{f}_{0}(*)$ for any $x \in \partial(I \times I)$, and $o\left(a_{g}\right)=0$. Replacing the $G$-isotopy $f$ between $f_{0}$ and $f_{1}$ by $g$, we can assume $o\left(a_{g}\right)=0$.

We now turn to the case $d=2$. If $V_{1}$ is isomorphic to $\oplus_{i} k_{i} \mathrm{~W}_{i}$, then $\operatorname{Mon}^{G}\left(V_{1}, 2 V_{1}\right)=\prod_{i} V_{2 k_{i}, k_{i}}^{\prime}\left(F_{i}\right)$ by Lemma 3.2. Note that $\pi_{1}\left(V_{2 k_{i}, k_{i}}^{\prime}\left(F_{i}\right)\right)$ is 0 beside the case $F_{i}=R$ and $k_{i}=1$. Let $J$ be the set of index $i$ 
such that $F_{i}=R$ and $k_{i}=1$. Let $p: \prod_{i} V_{2 k_{i}, k_{i}}^{\prime}\left(F_{i}\right) \rightarrow \prod_{i \in J} V_{2,1}(R)$ be the natural projection. Then $p_{*}: \pi_{1}\left(\prod_{i} V_{2 k_{i} k_{i}}^{\prime}\left(F_{i}\right)\right) \rightarrow \pi_{1}\left(\prod_{i \in J} V_{2,1}^{\prime}(R)\right)$ is isomorphic. Let $r: I \rightarrow \prod_{i \in J} V_{2,1}^{\prime}(R)$ be a continuous map defined by $r(t)=p \cdot \hat{f}_{t}(*)$. Since $\pi: \prod_{i \in J} G L(2, R) \rightarrow \prod_{i \in J} V_{2,1}^{\prime}$ is a product bundle, there exists a continuous map $\tilde{r}: I \rightarrow \prod_{i \in J} G L(2, R)$ such that $\pi \cdot \tilde{r}=r$ and $\tilde{r}(0)=\tilde{r}(1)$. Note that, for each $i \in J, G L(2, R)$ can be regarded as the automorphism group $A\left(2 W_{i}\right)$ of $G$-module $2 W_{i}$ whose element defines an equivariant linear diffeomorphism of $S^{\mathrm{w}}$. Let $g: S^{\mathrm{v}} \times I \rightarrow S^{\mathrm{w}}$ be a $G$-isotopy between $f_{0}$ and $f_{1}$ defined by $g_{t}=\tilde{r}(0) \cdot \tilde{r}(t)^{-1} \cdot f_{t}$. Since $\pi$ is identified with the natural map $\prod_{i \in J} A^{G}\left(2 W_{i}\right) \rightarrow \prod_{i \in J} \operatorname{Mon}^{G}\left(W_{i}, 2 W_{i}\right)$, $p \cdot \hat{g}_{t}(*)=p \cdot \hat{f}_{0}(*)$ and $o\left(g_{f}\right)=0$. Replacing the $G$-isotopy $f$ between $f_{0}$ and $f_{1}$ by $g$, we can assume $o\left(a_{f}\right)=0$.

Therefore we can assume that $a_{f}$ can be extended to $S^{n} \times I \times I$. Let $F: U \times I \times I \rightarrow N \times I$ be an equivariant map defined by $F(v, t, s)=$ $\left(a_{f}(q(v), t, s)(v), t\right)$, where $q: U \rightarrow S^{n}$ is the bundle projection. Then each $F(\cdot, t, s)$ is a $G$-imbedding, and $F_{0}(u, t)=\left(f_{t}(u), t\right)=\left(f_{t}^{\prime}(u), t\right)$ and $F_{1}(u, t)=\left(f_{0}(u), t\right)=\left(f_{0}^{\prime}(u), t\right)$ for $(u, t) \in U \times I$. By Lemma 1.2 we can assume that $F$ is a $G$-isotopy. In the same way as the proof of Proposition 2.3, we have a $G$-isotopy $h: S^{\mathrm{v}} \times I \rightarrow S^{\mathrm{w}}$ such that $h_{i}=f_{i}(i=0,1)$ and $h_{t}=f_{0}^{\prime}(0 \leqq t \leqq 1)$ on some invariant neighborhood of $S^{n}$. Therefore $\hat{h}_{t}=\hat{f}_{0}$ for each $t$, and this completes the proof of Proposition 3.3.

Remark. I don't know whether Proposition 3.3 is valid without the assumption $\pi_{n+1}\left(\operatorname{Mon}^{G}\left(V_{1}, d V_{1}\right)\right)=0$.

Now we shall assume $\pi_{n+1}\left(\operatorname{Mon}^{G}\left(V_{1}, d V_{1}\right)\right)=0$. Choose a continuous map $a_{2}: S^{n} \rightarrow O^{G}\left(V_{1}, d V_{1}\right)$, which represents an element $\lambda$, for each element $\lambda$ of $\pi_{n}\left(O^{G}\left(V_{1}, d V_{1}\right)\right)$. Let $A=\left\{a_{\lambda} ; \lambda \in \pi_{n}\left(O^{G}\left(V_{1}, d V_{1}\right)\right\}\right.$

Definition 3.4. Let $f_{i}: S^{\mathrm{v}} \rightarrow S^{\mathrm{W}}, i=0,1$, be $G$-imbeddings, which represent elements of $I_{1}\left(S^{v}, S^{\mathrm{w}}\right)$, such that $\hat{f}_{i}, i=0,1$, are elements of $A . f_{0}$ and $f_{1}$ are said to be equivalent if there exists a $G$-isotopy $f: S^{\mathrm{V}} \times I \rightarrow S^{\mathrm{w}}$ between $f_{0}$ and $f_{1}$ such that $\hat{f}_{t}=\hat{f}_{0}$ for $0 \leqq t \leqq 1$. Let 
$I_{2}\left(S^{v}, S^{w}\right)$ denote the set of equivalence classes of these $G$-imbeddings.

Corollary 3.5. If $\pi_{n+1}\left(\operatorname{Mon}^{c}\left(V_{1}, d V_{1}\right)\right)=0$, the natural map $i_{2}: I_{2}\left(S^{v}, S^{W}\right) \rightarrow I_{1}\left(S^{v}, S^{W}\right)$ is bijective.

Proof. Let $f: S^{v} \rightarrow S^{W}$ be a $G$-imbedding which represents an element of $I_{1}\left(S^{v}, S^{W}\right)$. By Lemma 3.2 $O^{G}\left(V_{1}, d V_{1}\right)$ is a deformation retract of $\operatorname{Mon}^{G}\left(V_{1}, d V_{1}\right)$. Therefore, by Proposition 3.1, we can assume that $f$ is an element of $A$, and $i_{2}$ is surjective. By Proposition $3.3, i_{2}$ is injective, and this completes the proof of Corollary 3.5.

\section{$\S 4$. Proof of Theorem $A$ and Theorem $B$}

In this section we shall prove that, if $G$ is a finite group and $\pi_{n+1}\left(\operatorname{Mon}^{G}\left(V_{1}, d V_{1}\right)\right)=0$, then the $G$-isotopy class of a $G$-imbedding $f: S^{v} \rightarrow S^{W}$ is determined by the homotopy class of the orbit map of $f \mid\left(S^{v}-U\right)$ relative to the boundary, where $U$ is an invariant open neighborhood of $S^{n}$. And, using the obstruction theory, we shall prove Theorem A and Theorem B.

In this section we shall assume that $G$ is a finite group and $\pi_{n+1}\left(\operatorname{Mon}^{G}\left(V_{1}, d V_{1}\right)\right)=0$. Let $f_{i}: S^{v} \rightarrow S^{w}, i=0,1$, be $G$-imbeddings which represent elements of $I_{2}\left(S^{v}, S^{w}\right)$. Let $U$ be an invariant open $\varepsilon$-tubular neighborhood of $S^{n}$ in $S^{v}$. We can choose a sufficiently small positive number $\varepsilon$ such that $f_{i}=f_{i}^{\prime}$ on $U$ and $f_{i}\left(S^{v}-U\right) \subset S^{W}-T$ for $i=0,1$. By Corollary 3.5, we have the following :

Lemma 4. 1. With the above notations, $f_{0}$ and $f_{1}$ are $G$-isotopic if and only if there exists a G-isotopy $f: S^{v} \times I \rightarrow S^{w}$ such that $f_{t}\left(S^{v}-U\right)$ is contained in $S^{\mathrm{W}}-T$ and $f_{t}=f_{0}, 0 \leqq t \leqq 1$, on $U$.

It is clear that free $G$-manifolds $S^{v}-U$ and $S^{W}-T$ are equivariant diffeomorphic to $S\left(V_{1}\right) \times D^{n+1}$ and $S\left(d V_{1}\right) \times D^{d_{n+k+1}}$ respectively. Let $L$ and $L^{\prime}$ denote the orbit spaces $S\left(V_{1}\right) / G$ and $S\left(d V_{1}\right) / G$ respectively. Then the orbit spaces $\left(S^{v}-U\right) / G$ and $\left(S^{W}-T\right) G$ are diffeomorphic to $L \times D^{n+1}$ and $L^{\prime} \times D^{d n+k+1}$ respectively. Let $\bar{f}_{i}: L \times D^{n+1} \rightarrow L^{\prime} \times D^{d_{n+k+1}}$, 
$i=0,1$, be imbeddings defined by the orbit maps of $f_{i} \mid\left(S^{v}-U\right)$.

Proposition 4. 2. With the above notations, $f_{0}$ and $f_{1}$ are $G$-isotopic if and only if $\bar{f}_{0}$ and $\bar{f}_{1}$ are homotopic relative to $L \times S^{n}$.

Proof. By Lemma 4.1, if $f_{0}$ and $f_{1}$ are $G$-isotopic, then $\bar{f}_{0}$ and $\bar{f}_{1}$ are homotopic relative to $L \times S^{n}$. Conversely if $\bar{f}_{0}$ and $\bar{f}_{1}$ are homotopic relative to $L \times S^{n}$, then $\bar{f}_{0}$ and $\bar{f}_{1}$ are isotopic relative to $L \times S^{n}$ because $\operatorname{dim}\left(L^{\prime} \times D^{d_{n+k+1}}\right)>2 \operatorname{dim}\left(L \times D^{n+1}\right)+1$. Since $G$ is a finite group, $\quad S^{v}-U \rightarrow\left(S^{v}-U\right) / G$ and $S^{w}-T \rightarrow\left(S^{w}-T\right) / G$ are covering spaces. By the covering homotopy property, there exists a $G$-isotopy $h_{t}: S^{v}-U \rightarrow S^{\mathbb{W}}-T(0 \leqq t \leqq 1)$ relative to $\partial\left(S^{v}-U\right)$ such that $h_{0}=f_{0}$ and $\bar{h}_{t}=\bar{f}_{t}$ for $0 \leqq t \leqq 1$. Since $h_{1}\left|\partial\left(S^{v}-U\right)=f_{1}\right| \partial\left(S^{v}-U\right)$ and $\bar{h}_{1}=\bar{f}_{1}$, by the property of the covering space, we have $h_{1}=f_{1}$ on $S^{v}-U$. Therefore $f_{0}$ and $f_{1}$ are $G$-isotopic and this completes the proof of Proposition 4.2.

Proof of Theorem A. Suppose that $V_{1}=\underset{i}{\oplus} k_{i} W_{i}$, where $W_{i}$ runs over the inequivalent irreducible real $G$-modules. If $q>2, M^{G}$ $\left(V_{1}, d V_{1}\right)=\prod_{i} V_{d k_{i}, k_{i}}^{\prime}(C)$ by Lemma 3.2. Since $V_{d k_{i}, k_{i}}^{\prime}(C)$ is $2(d-1) k_{i}$ connected, $\pi_{n+1}\left(\operatorname{Mon}^{G}\left(V_{1}, d V_{1}\right)\right)=0$ if $d \geqq(n+3) / 2$. If $q=2, V_{1}=$ $(m-n) W_{1}$ and $\operatorname{Mon}^{G}\left(V_{1}, d V_{1}\right)=V_{d(m-n), m-n}^{\prime}(R)$, where $W_{1}$ is the nontrivial 1-dimensional real representation of $Z_{2}$. Since $V_{d(m-n), m-n}^{\prime}(R)$ is $(d-1)(m-n)-1$-connected, $\pi_{n+1}\left(\operatorname{Mon}^{G}\left(V_{1}, d V_{1}\right)\right)=0$ if $d \geqq(m+2) /$ $(m-n)$. Therefore, combining Corollary 2.5 and Corollary 3.5, the set $I\left(S^{v}, S^{w}\right)$ can be identified with the set $I_{2}\left(S^{v}, S^{w}\right)$. Let $f_{S}: S^{v} \rightarrow S^{w}$ be the standard imbedding. Let $f: S^{v} \rightarrow S^{W}$ be a $G$-imbedding which represents an element of $I_{2}\left(S^{v}, S^{W}\right)$. Since $\pi_{n}\left(\operatorname{Mon}^{G}\left(V_{1}, d V_{1}\right)\right)=0$, we can assume $\hat{f}=\hat{f}_{s}$. With the notation of Proposition 4.2, $f$ and $f_{s}$ are $G$-isotopic if and only if $\bar{f}$ and $\bar{f}_{S}$ are homotopic relative to $S^{n} \times L$.

Note that $L$ (resp. $L^{\prime}$ ) is an $m-n-1$ (resp. $\left.d(m-n)-1\right)$-dimensional lens space or real projective space. Since $d \geqq(m+2) /(m-1)$, $\pi_{i}\left(L^{\prime} \times D^{d_{n+k+1}}\right)=0$ for $2 \leqq i \leqq m$. By the obstruction theory of P. Olum [5, Theorem 9.10 and Theorem 16.5], $\bar{f}$ and $\bar{f}_{S}$ are homotopic relative to $L \times S^{n}$. This completes the proof of Theorem A. 
Proof of Theorem B. By Lemma 3.2, $\operatorname{Mon}^{G}\left(V_{1}, d V_{1}\right)=V_{d(m-n) / 2,(m-n) / 2}^{\prime}$ $(C)$. Thus $\operatorname{Mon}^{G}\left(V_{1}, d V_{1}\right)$ is $(d-1)(m-n)$-connected, and if $d \geqq(m+1) /(m-n), \pi_{n+1}\left(\operatorname{Mon}^{G}\left(V_{1}, d V_{1}\right)\right)=0$. Combining Corollary 2.5 and Corollary 3.5, the set $I\left(S^{v}, S^{\mathrm{W}}\right)$ can be identified with $I_{2}\left(S^{v}, S^{\mathrm{W}}\right)$.

Let $f: S^{v} \rightarrow S^{W}$ be a $G$-imbedding which represents an element of $I_{2}\left(S^{v}, S^{w}\right)$. Similarly as the proof of Theorem A, we can assume $\bar{f}\left|L \times S^{n}=\bar{f}_{S}\right| L \times S^{n}$, and in the case of $d>(m+1) /(m-n), f$ is $G$ isotopic to the standard imbedding $f_{s}$.

Now consider the case of $d=(m+1) /(m-n)$. Since $H^{i}\left(L \times D^{n+1}\right.$, $\left.L \times S^{n} ; \pi_{i}\left(L^{\prime} \times D^{d_{n+k+1}}\right)\right)=H^{i-n-1}\left(L ; \pi_{i}\left(L^{\prime}\right)\right)=0$ for $i<m$ and $H^{i-1}$ $\left(L \times D^{n+1}, L \times S^{n} ; \pi_{i}\left(L^{\prime} \times D^{d n+k+1}\right)\right)=H^{i-n-2}\left(L ; \pi_{i}\left(L^{\prime}\right)\right)=0$ for $i<m$, by the obstruction theory, the homotopy classes of maps $\bar{f}: L \times D^{n+1} \rightarrow$ $L^{\prime} \times D^{d_{n+k+1}}$ relative to $L \times S^{n}$ are in one to one correspondence with the elements of

$$
H^{m}\left(L \times D^{n+1}, L \times S^{n} ; \pi_{m}\left(L^{\prime} \times D^{d_{n+k+1}}\right)\right)=\pi_{m}\left(L^{\prime}\right) .
$$

Since $\operatorname{dim} L^{\prime}=d(m-n)-1=m$ in the case of $d=(m+1) /(m-n)$, by Proposition 4.2, we have $I\left(S^{v}, S^{w}\right)=Z$. This completes the proof of Theorem B.

Remark. Suppose that $V_{1}=\bigoplus_{i} k_{i} W_{i}$ and $k_{i} \geqq 3$ for each $i$ if $\operatorname{dim}$ $\operatorname{Hom}^{G}\left(W_{\imath}, W_{i}\right)=1$, where $W_{\imath}$ runs over the inequivalent irreducible real $G$-modules. Then Theorem A is valid when $G$ is a finite group.

\section{References}

[1] Bredon, G., Introduction to Compact Transformation Groups, Academic Press, New York and London, 1972.

[2] Dax, J., Étude homotopique des espaces de plongements, Ann. Sci. Ecole Norm. Sup., 5 (1972), 303-377

[3] Hirsch, M, Differential Topology, Springer-Verlag, Berlin-Heidelberg New York, 1976.

[4] Munkres, J., Elementary Differential Topology, Princeton Univ. Press, 1966.

[5] Olum, P., Obstruction to extensions and homotopies, Ann. of Math., 52 (1950), 1-50.

[6] Serre, J.-P., Représentations Linéaires des Groupes Finis, Hermann S. A., Paris, 1971.

[7] Wasserman, A., Equivariant differential topology, Topology, 8 (1969), 127-150. 